\title{
Accuracy of PfHRP2 versus Pf-pLDH antigen detection by malaria rapid diagnostic tests in hospitalized children in a seasonal hyperendemic malaria transmission area in Burkina Faso
}

\author{
Jessica Maltha ${ }^{1,2^{*}}$, Issa Guiraud ${ }^{3}$, Palpouguini Lompo ${ }^{3}$, Bérenger Kaboré ${ }^{3}$, Philippe Gillet ${ }^{1}$, Chris Van Geet ${ }^{2,4}$,
} Halidou Tinto $^{3}$ and Jan Jacobs ${ }^{1}$

\begin{abstract}
Background: In most sub-Saharan African countries malaria rapid diagnostic tests (RDTs) are now used for the diagnosis of malaria. Most RDTs used detect Plasmodium falciparum histidine-rich protein-2 (PfHRP2), though P. falciparum-specific parasite lactate dehydrogenase (Pf-pLDH)-detecting RDTs may have advantages over PFHRP2-detecting RDTs. Only few data are available on the use of RDTs in severe illness and the present study compared Pf-pLDH to PfHRP2-detection.

Methods: Hospitalized children aged one month to 14 years presenting with fever or severe illness were included over one year. Venous blood samples were drawn for malaria diagnosis (microscopy and RDT), culture and complete blood count. Leftovers were stored at $-80^{\circ} \mathrm{C}$ and used for additional RDT analysis and PCR. An RDT targeting both PfHRP2 and Pf-pLDH was performed on all samples for direct comparison of diagnostic accuracy with microscopy as reference method. PCR was performed to explore false-positive RDT results.

Results: In 376 of 694 (54.2\%) included children, malaria was microscopically confirmed. Sensitivity, specificity, positive predictive value (PPV) and negative predictive value were 100.0, 70.9, 69.4 and $100.0 \%$, respectively for PfHRP2-detection and 98.7, 94.0, 91.6 and 99.1\%, respectively for Pf-pLDH-detection. Specificity and PPV were significantly lower for PfHRP2-detection $(p<0.001)$. For both detection antigens, specificity was lowest for children one to five years and in the rainy season. PPV for both antigens was highest in the rainy season, because of higher malaria prevalence. False positive PfHRP2 results were associated with prior anti-malarial treatment and positive PCR results (98/114 (86.0\%) samples tested).

Conclusion: Among children presenting with severe febrile illness in a seasonal hyperendemic malaria transmission area, the present study observed similar sensitivity but lower specificity and PPV of PFHRP2 compared to Pf-pLDH-detection. Further studies should assess the diagnostic accuracy and safety of an appropriate Pf-pLDH-detecting RDT in field settings and if satisfying, replacement of PfHRP2 by Pf-pLDH-detecting RDTs should be considered.
\end{abstract}

\footnotetext{
* Correspondence: jessicamaltha@gmail.com

'Department of Clinical Sciences, Institute of Tropical Medicine,

Nationalestraat 155, B 2000, Antwerp, Belgium

${ }^{2}$ Centre for Molecular and Vascular Biology, University of Leuven, Leuven,

Belgium

Full list of author information is available at the end of the article
} waiver (http://creativecommons.org/publicdomain/zero/1.0/) applies to the data made available in this article, unless otherwise stated. 


\section{Background}

Malaria rapid diagnostic tests (RDTs) are currently rolled out in sub-Saharan Africa to fulfill the need of parasite based diagnosis e.g. the parasitological confirmation of malaria before start of treatment [1]. The operational characteristics of RDTs have been extensively evaluated for uncomplicated malaria [2] and the parasite-based diagnosis strategy has proven to be safe in uncomplicated malaria [3]. In contrast, only a few studies addressed the use of RDTs in children presenting with severe illness. Those performed reported low specificity of Plasmodium falciparum histidine-rich protein-2 (PfHRP2)-detecting RDTs $[4,5]$, which is most probably due to PfHRP2 persistence after clearance of infection [6]. An alternative would be an RDT detecting P. falciparum-specific parasite lactate dehydrogenase $(P f-\mathrm{pLDH})$, which is more rapidly cleared from the bloodstream, but lower sensitivities compared to PfHRP2 have been reported [4]. However recent evaluations of other $P f$-pLDH-detecting RDT products have shown better performance, also at low parasite densities [7-9]. The RDT used by the national malaria control programme of Burkina Faso detects PfHRP2, which is recommended by the World Health Organization (WHO) [10] and used in most sub-Saharan African countries. The current diagnostic algorithm in Burkina Faso recommends treatment of malaria in case of a positive test and search for other causes of disease when negative [11], but does not differentiate between severe and non-severe disease. The aim of this study was to compare Pf-pLDH to PfHRP2-detecting RDTs in children presenting with severe febrile illness in a seasonal, hyperendemic, malaria transmission area.

\section{Methods}

\section{Study site and population}

A one-year survey (July 2012-2013) to assess proportions and incidence rates of invasive bacterial infections and severe malaria was performed in a rural area in the centre-west region of Burkina Faso. In this region there is seasonal hyperendemic malaria transmission and the estimated under-five mortality in 2010 was $142 / 1,000$ live births [12]. Details of the study have been published elsewhere [13]. In summary, children $(<15$ years $)$ presenting with axillary temperature $\geq 38.0{ }^{\circ} \mathrm{C}$ and/or clinical signs of severe illness who were admitted to the hospital or health centre were enrolled. Signs of severe illness included convulsions, altered consciousness, prostration, respiratory distress, shock, hypothermia and severe malnutrition. For the present study, children $<$ one month of age were not considered.

\section{Sample collection}

In all children enrolled blood culture was performed and ethylene diamine tetra-acetic acid (EDTA)-anticoagulated venous blood samples were drawn for malaria diagnosis (both microscopy and RDT). Laboratory analysis was performed in the clinical research unit of Nanoro (CRUN), located on the compound of the district hospital. Leftovers of EDTA blood samples were stored at $80{ }^{\circ} \mathrm{C}$ within a maximum of two hours after sampling until further analysis. Medical history, including previous anti-malarial treatment, and clinical examination were registered on standardized forms by trained study staff.

\section{Laboratory procedures}

Thick blood films (TBF) were stained with Giemsa and assessed for the presence of Plasmodium parasites according to standard procedures [14]. Parasite density was expressed as asexual parasites per $\mu \mathrm{l}$ using the patient's white blood cell (WBC) count. TBF was considered negative if no parasites were seen on 100 fields. Every slide was read by two experienced microscopists blinded to each other's results and in case of discrepant results (positive $v s$ negative, different Plasmodium species, difference in parasite density $>\log 10$ or ratio $>2$ in case of parasite density $\leq 400 / \mu \mathrm{l}$ and $>400 / \mu \mathrm{l}$, respectively) by a third experienced microscopist.

The RDT SD Bioline Malaria Antigen P.f (Standard Diagnostics, Hagal-Dong, Korea), further referred to as SD50 (LOT 082160), is the RDT recommended by the national malaria control programme of Burkina Faso and detects the protein PfHRP2. SD50 was performed on EDTA blood samples by trained CRUN laboratory staff before slides were read and within a maximum of two hours after sampling. Blood culture work-up and cerebrospinal fluid analysis was performed according to standard microbiological procedures as described previously [13].

\section{Malaria rapid diagnostic test evaluated}

The RDT SD Bioline Malaria Antigen P.f (HRP2/pLDH) (Standard Diagnostics, Hagal-Dong, Korea), further referred to as SD90 (LOT RDT12002), is a three-band test consisting of a control line and two test lines targeting PfHRP2 and Pf-pLDH, respectively. Good performance was reported in previous evaluations $[15,16]$. SD90 was performed on EDTA blood samples according to the manufacturer's instructions except for replacement of the transfer device by a micropipette. From February to July $(\mathrm{n}=276)$, SD90 was performed on fresh samples side-to-side to SD50 by CRUN laboratory staff. For the remaining samples $(n=420)$ SD90 was performed on stored samples at the end of the study period by the investigator.

In case of absence of the control line the test was considered invalid and repeated. Test line intensities were scored as negative, faint, weak, medium, or strong compared to the control line by a single observer who was 
blinded to the result of microscopy. After reading, photographs were taken.

\section{Monitoring and quality control}

A selection of slides (5\%) was sent to the Institute of Tropical Medicine (ITM) and again read by an expert microscopist whose results were considered conclusive. SD50 and SD90 were ordered at ITM Belgium and shipped to Burkina Faso where they were stored in a temperaturecontrolled room. The actual kit in use was stored in the parasitology laboratory. Temperature and humidity during shipment and storage were monitored using loggers (Ebro Electronic GmBH, Ingolstadt, Germany).

For discordant results between either SD50 or SD90 and microscopy, samples were retrieved from $-80{ }^{\circ} \mathrm{C}$ storage and both RDTs were repeated by the investigator blinded to microscopy results. The result of the repeat testing was considered for analysis in case the first result was performed by CRUN staff. If both the first and repeat testing was performed by the investigator, the first result was considered. Photographs taken were verified to exclude clerical errors.

In order to compare PfHRP2 results of SD50 and SD90, the RDTs were performed side to side on $10 \%$ randomly selected stored samples. For SD50, these 10\% of stored samples were also compared to results obtained when prospectively performed on fresh samples.

\section{Additional analysis: polymerase chain reaction (PCR)}

Real-time polymerase chain reaction (PCR) was performed in case of discordant results between microscopy and RDT. DNA was extracted from $200 \mathrm{ml}$ whole blood using QIAamp DNA blood Mini kit (QIAGEN, Venlo, The Netherlands) or from TBF if needed [17]. DNA was amplified by a species-specific $18 \mathrm{~S}$ rRNA real-time PCR (P. falciparum/Plasmodium vivax [18]), the Plasmodium ovale/Plasmodium malariae duplex was run simultaneously to confirm microscopically identified non-falciparum species.

\section{Data management, definitions and analysis}

Data were double-entered in Epi info software (version 3.5.3). Statistical analysis was done with Stata 11 (Stata Corp, College Station, TX, USA). For the purpose of this study, microscopy was considered as the gold standard. Samples with asexual P. falciparum parasites seen on TBF (irrespective of parasite density and either as mono-infection or as mixed infection with $P$. ovale or $P$. malariae) were categorized as $P$. falciparum positive. The remaining samples, including samples with pure $P$. falciparum gametocytaemia, $P$. ovale or $P$. malariae as well as those with no parasites seen, were categorized as $P$. falciparum negative. For SD90, sensitivity and specificity were calculated for both PfHRP2 and Pf-pLDH test lines. A visible test line in case of $P$. falciparum positive samples was considered true positive, no visible test line false negative. For $P$. falciparum negative samples, the absence of a visible test line was labelled as true negative, a visible line was labelled as false positive. Sensitivity, specificity and predictive values were calculated by age group and season and expressed with 95\% CI. Differences were assessed for statistical significance using the Chi-square test, or Fisher exact test when appropriate, in case of independent data (e.g., comparison between the seasons) and with the McNemar test or paired proportion test for dependent data (PfHRP2 vs Pf-pLDH).

\section{Ethical issues}

The study was approved by the ethical committee of Burkina Faso and the University Hospital of Antwerp and by the institutional review board of ITM. Written informed consent was obtained from the parent or guardian of each child included.

\section{Results}

\section{Study population and malaria microscopy results}

During the one-year study period, 696 children aged one month to 14 years were included [13]. For two children (both $P$. falciparum positive with parasite densities of $62 / \mu \mathrm{l}$ and $35,194 / \mu \mathrm{l}$, respectively), there was evidence of a sample switch during storage, both samples were excluded from analysis. The final collection consisted of 694 samples.

Demographic data of participants during the different seasons are shown in Table 1. Seasons were divided into rainy season (July to October, monthly microscopy positivity rate $67.9-87.9 \%$ ), post-rainy season (November to February, monthly microscopy positivity rate $31.4-40.0 \%$ ) and hot dry season (March to June, monthly microscopy positivity rate $2.9-17.2 \%)$.

In 376 (54.0\%) children, samples were $P$. falciparum positive, five of them had a mixed infection with $P$. malariae $(\mathrm{n}=3)$ and $P$. ovale $(\mathrm{n}=2)$. Median $P$. falciparum parasite density was $43,231.5 / \mu \mathrm{l}(25-702,500)$. Among the $P$. falciparum negative samples $(318 / 694,45.8 \%)$, two had $P$. ovale infection and 13 had pure $P$. falciparum gametocytaemia.

\section{Diagnostic accuracy of PfHRP2 and Pf-pLDH}

No invalid results were observed for SD90. PfHRP2 and $P f$-pLDH positivity rates are shown in Figure 1. Among the $P$. falciparum-positive samples, $P f \mathrm{HRP} 2$ was positive in all samples while $P f$-pLDH missed one (parasite density $62 / \mu$ l, Table 2 ), resulting in an overall sensitivity of 100 and $99.5 \%$ for PfHRP2 and $P f-\mathrm{pLDH}$, respectively ( $p=$ 1.0). Among the $P$. falciparum-negative samples, there were 139 and 29 false-positive PfHRP2 and Pf-pLDH lines, respectively (Figure 2), corresponding to specificities of 
Table 1 Demographic profile and diagnosis of children included during the different seasons

\begin{tabular}{|c|c|c|c|c|}
\hline & All year & Rainy season & Post-rainy season & Dry season \\
\hline Number & 694 & 398 & 151 & 145 \\
\hline Age, median months (IQR) & $20(11-37)$ & $21(12-37)$ & $16(9-35)$ & $17(9-39)$ \\
\hline Female sex, n (\%) & $310(44.7)$ & $176(44.2)$ & $79(52.3)$ & $55(37.9)$ \\
\hline Prior antimalarial treatment, $\mathrm{n}(\%)$ & $302(43.5)$ & $156(39.2)$ & $87(57.6)$ & $59(40.7)$ \\
\hline Microscopy Pf positive & $376(54.2)$ & $304(76.4)$ & $55(36.4)$ & $17(11.7)$ \\
\hline Pf parasite density/ $\mu \mathrm{l}$, median & 42,331 & $49,962.5$ & 18,256 & 7,549 \\
\hline Pf parasite density/ $\mu$, range & $25-702,500$ & $62-702,500$ & $99-259,685$ & $25-112,465$ \\
\hline Blood culture positive, n (\%) & $60(8.7 \%)$ & $19(4.8)$ & $27(17.9)$ & $14(9.7)$ \\
\hline Confirmed meningitis, n (\%) & $6(0.9)$ & $1(0.3)$ & $2(1.3)$ & $3(2.1)$ \\
\hline Co-infections & $7(1.0)$ & $4(1.0)$ & $3(2.0)$ & 0 \\
\hline
\end{tabular}

$\mathrm{IQR}=$ interquartile range, $\mathrm{n}=$ number, $P f=P$. falciparum.

56.3 and $90.9 \%$, respectively ( $<<0.001)$. In $25(7.9 \%)$ P. falciparum-negative samples both test lines were visible: they included eight samples with pure gametocytaemia. PfHRP2 was positive in an additional 114 samples, including another four samples with pure gametocytaemia. False positive $P f$-pLDH test lines in the absence of PfHRP2 lines occurred in four samples of which two were $P$. ovale infection and one pure gametocytaemia.

Overall, positive predictive value (PPV) for PfHRP2detection (73.0\%) was significantly lower compared to $P f$-pLDH detection $(92.8 \%, p<0.001)$ while negative predictive values (NPV) were similar (Table 3 ).

\section{PfHRP2 and Pf-pLDH performance by age group}

PfHRP2 specificity was significantly lower among children aged one to five years $(39.6 \%)$ compared to children $<$ one year $(70.9 \%)$ or $>$ five years $(68.4 \%, p<0.001$ for both), for $P f$-pLDH differences among age groups were not significant $(p=0.053$ and $p=0.134$, respectively, Table 4). As the slide-positivity rate was highest among children one to five years of age, PPV for PfHRP2 in this age group did not differ significantly from the others despite lower specificity $(p=0.428$ and $p=0.255)$.

\section{PfHRP2 and Pf-pLDH seasonal performance}

Sensitivity of PfHRP2 and Pf-pLDH detection did not differ throughout the year (Table 4). PfHRP2 specificity was significantly higher in the hot dry season (78.9\%) compared to the rainy and post-rainy season (35.1 and $46.9 \%$, respectively, both $p<0.001$ ). As slide positivityrate was highest in the rainy season, PPV of PfHRP2 was highest in the rainy season (83.3\%), despite lowest specificity, and decreased to 51.9 and $38.6 \%$ in the other two seasons. Specificity of $P f$-pLDH detection was in all

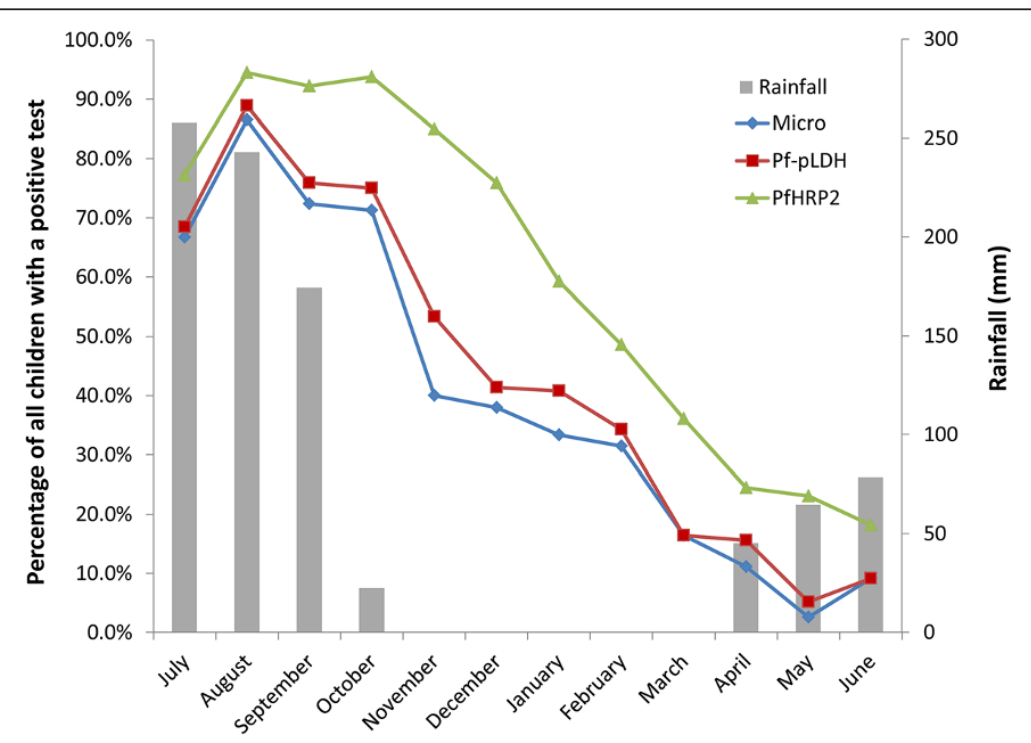

Figure 1 Microscopy, PfHRP2 and Pf-pLDH positivity rate by month. Micro = microscopy positivity rate, PfHRP2 = positivity rate of PfHRP2, Pf-pLDH = positivity rate of Pf-pLDH. 
Table 2 PfHRP2 and Pf-pLDH results according to parasite density

\begin{tabular}{|c|c|c|c|c|c|}
\hline \multirow[b]{2}{*}{ Microscopy } & \multirow[b]{2}{*}{ Number } & \multicolumn{2}{|c|}{ PfHRP2 pos } & \multicolumn{2}{|c|}{ PfHRP2 neg } \\
\hline & & $\begin{array}{l}\text { Pf-pLDH } \\
\text { pos }\end{array}$ & $\begin{array}{c}\text { Pf-pLDH } \\
\text { neg }\end{array}$ & $\begin{array}{c}\text { Pf-pLDH } \\
\text { pos }\end{array}$ & $\begin{array}{c}\text { Pf-pLDH } \\
\text { neg }\end{array}$ \\
\hline $1-100$ & 3 & 2 & 1 & & \\
\hline $101-1,000$ & 25 & 25 & & & \\
\hline $1,001-10,000$ & 66 & 66 & & & \\
\hline $10,001-100,000$ & 199 & 199 & & & \\
\hline$>100,000$ & 83 & 83 & & & \\
\hline pure gametocytemia & 13 & 8 & 4 & 1 & \\
\hline Microscopy negative & 303 & 17 & 110 & 1 & 175 \\
\hline P. ovale & 2 & & & 2 & \\
\hline Total & 694 & 400 & 115 & 4 & 175 \\
\hline
\end{tabular}

Neg = negative, pos = positive, $P f \mathrm{HRP} 2=P$. falciparum Histidine-rich protein-2, $P f$ - $\mathrm{pLDH}=P$. falciparum-specific parasite lactate dehydrogenase.

seasons higher compared to PfHRP2 (Table 4, $p<0.001$ for each season). Also for Pf-pLDH-detection PPV was highest in the rainy season, although differences were smaller: $95.6 \%$ in the rainy season compared to $82.1 \%$ $(p<0.001)$ and $85.0 \%(p=0.071)$ in the post-rainy and hot dry season, respectively.

\section{RDT positivity among children with invasive bacterial infections}

Microscopy was positive in eight out of 64 (12.5\%) children with invasive bacterial infections (IBI), PfHRP2 and Pf-pLDH were positive in 33/64 (51.6\%) and 12/64 (18.8\%), respectively. As described previously [13] non- typhoid Salmonella spp. (NTS) were most frequently isolated from blood culture (21/60, 35.0\%). PfHRP2 was positive among $17 / 21$ (81.0\%) NTS, which was significantly more frequent compared to $P f$-pLDH $(6 / 21,28.6 \%$, $p=0.002)$ and microscopy $(2 / 21,9.5 \%, p<0.001)$.

\section{RDT line intensity}

For $P f$-pLDH, $2.4 \%$ (nine/375) of true positive test lines was of faint intensity, for PfHRP2 this was $0.5 \%$ (two/ $376)$. For the $P$. falciparum positive samples, the PfHRP2 test line compared to the corresponding Pf-pLDH test line for the same sample was of stronger and weaker intensity in 179/376 (47.6\%) and 31/376 (8.2\%) samples, respectively. For seven samples with high parasite density $(82,080-392,535 / \mu \mathrm{l})$ PfHRP2 was of weak intensity while $P f$-pLDH was of strong intensity. Among the false positive test lines (excluding pure gametocytaemia), two/ 20 (10\%) Pf-pLDH and 67/126 (53.2\%) PfHRP2 lines were of medium or strong intensity.

\section{RDT results of retesting and quality control}

During the side-to-side comparison of PfHRP2 of SD50 and SD90, no discordances between positive and negative results were observed and differences in line intensity were limited to one category. When comparing results of SD50 performed on fresh and stored samples, no differences in positive/negative results nor major differences in line intensities (more than one category) were observed, except for one originally strong result which was faint on repeat testing but for which clerical error could not be excluded.

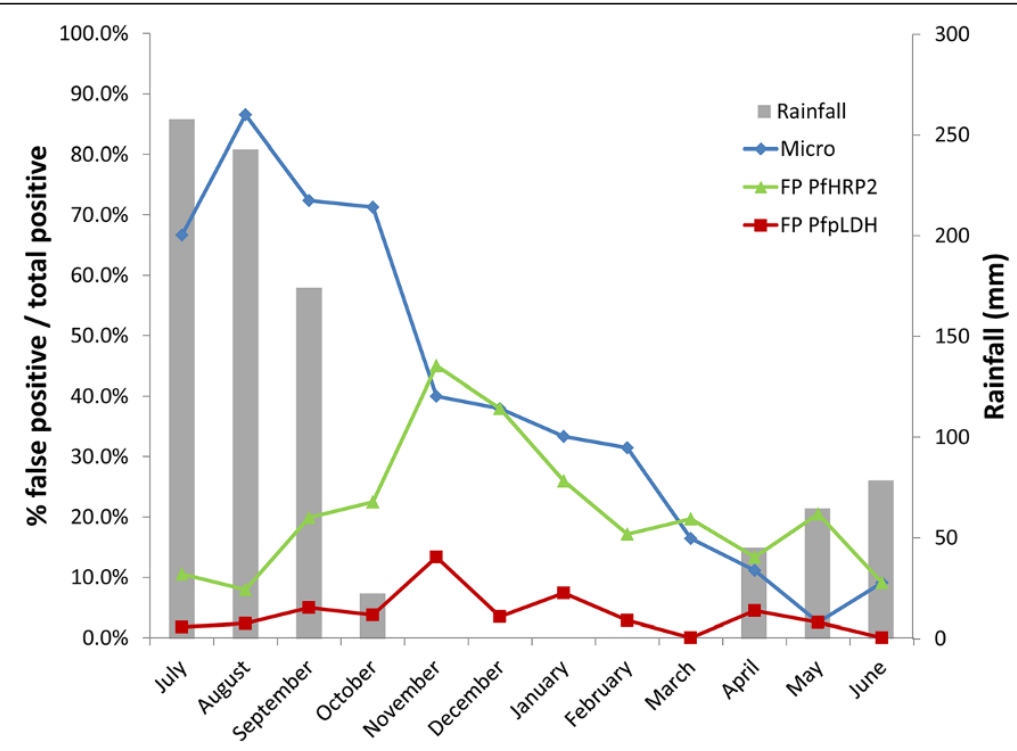

Figure 2 False positive rapid diagnostic test results per month compared to microscopy positivity rate. Micro = microscopy positivity rate among all children included per month, FP PfHRP2 $=\%$ false positive PfHRP2 results among total positive PfHRP2 results, FP PfpLDH $=\%$ false positive Pf-pLDH results among total positive Pf-pLDH results. 
Table 3 Diagnostic accuracy of PfHRP2- compared to Pf-pLDH-detection

\begin{tabular}{lllr}
\hline & PfHRP2 & Pf-pLDH & $\boldsymbol{p}$-value \\
\hline RDT pos, n (\%) & $515(74.2)$ & $404(54.2)$ & \\
SE $(95 \%$ Cl) & $100.0(94.7-100.0)$ & $98.7(93.5-99.9)$ & 1.0 \\
Sp $(95 \%$ Cl) & $70.9(67.4-70.9)$ & $94.0(90.6-94.8)$ & $<0.001$ \\
PPV (95\% Cl) & $69.4(65.7-69.4)$ & $91.6(86.8-92.7)$ & $<0.001$ \\
NPV (95\% Cl) & $100.0(95.1-100.0)$ & $99.1(95.5-100.0)$ & 1.0 \\
\hline
\end{tabular}

$\mathrm{N}=$ number, $\mathrm{NPV}=$ negative predictive value, $P A \mathrm{HPP} 2=P$. falciparum Histidine-rich protein-2, $P f$-pLDH $=P$. falciparum-specific parasite lactate dehydrogenase pos = positive, $\mathrm{PPV}=$ positive, predictive value $\mathrm{SE}=$ sensitivity, $\mathrm{Sp}=$ specificity.

\section{Further analysis of the false positive samples}

In 139/318 P. falciparum-negative samples the PfHRP2 line was visible, including 12 samples with pure gametocytaemia. For the latter, ten/12 were obtained in children reporting previous anti-malarial treatment and eight $/ 12$ had a visible $P f$-pLDH line. Figure 3 summarizes the results of PCR, $P f$-pLDH and previous anti-malarial treatment for the false positive PfHRP2 results (excluding pure gametocytaemia) for which PCR was performed $(\mathrm{n}=114)$ : $P f$-pLDH was positive in $16(14.0 \%)$ samples, PCR in $98(86.0 \%)$ and previous anti-malarial treatment was reported in 75 (65.8\%). For five samples, none of the aforementioned items was positive.

Among both PfHRP2 and Pf-pLDH false positive results, report of previous anti-malarial treatment was significantly higher (66.2 and $86.2 \%$, respectively) compared to microscopy positive samples $(33.3 \%, p<0.001$ for both).

\section{Discussion}

The present study assessed the diagnostic accuracy of $P f H R P 2$ compared to Pf-pLDH antigen detection in children presenting with severe febrile illness in a rural area with seasonal malaria transmission. Both antigens had excellent sensitivity and similar negative predictive values, but PfHRP2 had a lower specificity resulting in a significantly lower positive predictive value. Specificity was lowest in the rainy season, but due to the high malaria prevalence PPV was highest in the rainy season. The majority of false-positive PfHRP2 lines were PCR positive and/or reported recent anti-malarial treatment, part of them were also $P f$-pLDH positive or had pure gametocytaemia.

\section{Limitations}

A number of limitations need to be considered. First, although the present study allowed reliable comparison between $P f$-pLDH and PfHRP2-detection, which was the study objective, the RDT evaluated is not a format that is likely to be used in field settings. However, the simultaneous side-to-side testing of SD90 and SD50 showed similar results, therefore data about PfHRP2 performance can probably be extrapolated to the actual situation in Burkina Faso. Second, RDTs were performed and interpreted by an experienced investigator using a calibrated pipette, which may have generated higher sensitivities than would have been obtained under field conditions. Furthermore, 60\% of SD90 was performed on stored samples, although samples had been stored for

Table 4 Diagnostic accuracy of PfHRP2 versus Pf-pLDH by age group and by season

\begin{tabular}{|c|c|c|c|c|c|c|c|c|c|c|}
\hline & \multirow[t]{2}{*}{$\mathrm{Nr}$} & \multirow{2}{*}{$\begin{array}{l}\text { Slide Pf } \\
\text { pos (\%) }\end{array}$} & \multicolumn{4}{|c|}{ PfHRP2 } & \multicolumn{4}{|c|}{$P f$-pLDH } \\
\hline & & & $\begin{array}{l}\text { SE } \\
(95 \% \text { C.I.) }\end{array}$ & $\begin{array}{l}\text { Sp } \\
(95 \% \text { C.I. })\end{array}$ & $\begin{array}{l}\text { PPV } \\
(95 \% \text { C.I.) }\end{array}$ & $\begin{array}{l}\text { NPV } \\
(95 \% \text { C.I.) }\end{array}$ & $\begin{array}{l}\text { SE } \\
(95 \% \text { C.I.) }\end{array}$ & $\begin{array}{l}\text { Sp } \\
(95 \% \text { C.I. })\end{array}$ & $\begin{array}{l}\text { PPV } \\
\text { (95\% C.I.) }\end{array}$ & $\begin{array}{l}\text { NPV } \\
(95 \% \text { C.I.) }\end{array}$ \\
\hline \multicolumn{11}{|l|}{ Age (m) } \\
\hline \multirow[t]{2}{*}{$1-11$} & 194 & 39.7 & 100.0 & 70.9 & 69.4 & 100.0 & 98.7 & 94.0 & 91.6 & 99.1 \\
\hline & & & $94.7-100.0$ & $67.4-70.9$ & $65.7-69.4$ & $95.1-100.0$ & 93.5-99.9 & $90.6-94.8$ & 86.8-92.7 & $95.5-100.0$ \\
\hline \multirow[t]{2}{*}{$12-59$} & 401 & 64.1 & 100.0 & 39.6 & 74.7 & 100.0 & 100.0 & 86.8 & 93.1 & 100.0 \\
\hline & & & $98.3-100.0$ & $36.6-39.6$ & 73.5-74.7 & $92.5-100.0$ & $98.4-100.0$ & $84.0-86.8$ & $91.6-93.1$ & $96.7-100.0$ \\
\hline \multirow[t]{2}{*}{$\geq 60$} & 99 & 42.4 & 100.0 & 68.4 & 70.0 & 100.0 & 100.0 & 94.7 & 93.3 & 100.0 \\
\hline & & & $91.1-100.0$ & $61.9-68.4$ & $63.8-70.0$ & $90.4-100.0$ & $92.3-100.0$ & $89.1-94.7$ & $86.2-93.3$ & $94.0-100.0$ \\
\hline \multicolumn{11}{|l|}{ Season } \\
\hline \multirow[t]{2}{*}{ Rainy } & 398 & 76.4 & 100.0 & 35.1 & 83.3 & 100.0 & 99.7 & 85.1 & 95.6 & 98.8 \\
\hline & & & $98.7-100.0$ & $30.8-35.1$ & $82.2-83.3$ & $87.6-100.0$ & $98.2-100.0$ & $80.5-86.1$ & $94.2-95.9$ & 93.4-99.9 \\
\hline \multirow[t]{2}{*}{ Post-rainy } & 151 & 36.4 & 100.0 & 46.9 & 51.9 & 100.0 & 100.0 & 87.5 & 82.1 & 100.0 \\
\hline & & & $92.7-100.0$ & $42.7-46.9$ & 48.1-51.9 & $91.1-100.0$ & $93.2-100.0$ & $83.6-87.5$ & $76.5-82.1$ & $95.5-100.0$ \\
\hline \multirow[t]{2}{*}{ Hot dry } & 145 & 11.7 & 100.0 & 78.9 & 38.6 & 100.0 & 100.0 & 97.7 & 85.0 & 100.0 \\
\hline & & & 78.7-100.0 & $76.1-78.9$ & $30.4-38.6$ & $96.4-100.0$ & 81.7-100.0 & $95.2-97.7$ & $69.5-85.0$ & $97.5-100.0$ \\
\hline
\end{tabular}

$\mathrm{Pf}=P$. falciparum, $\mathrm{PfHRP2}=P$. falciparum Histidine-rich protein-2, $P f$-pLDH $=P$. falciparum-specific parasite lactate dehydrogenase, pos $=$ positive, $\mathrm{SE}=$ sensitivity, $\mathrm{Sp}=$ specificity, $\mathrm{PPV}=$ positive predictive value, $\mathrm{NPV}=$ negative predictive value. 


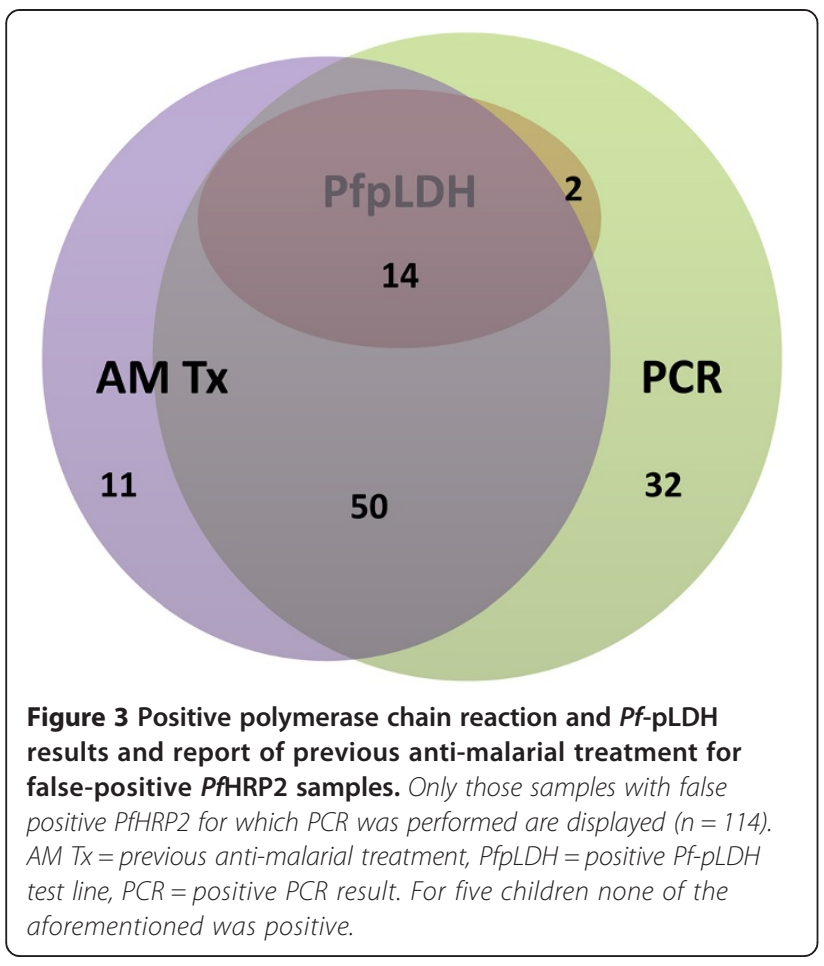

a maximum of one year and had not been thawed prior to testing; in addition, repeat testing on a subset of samples showed no difference in results when performed on fresh or stored samples.

\section{Sensitivity}

So far, only two studies have evaluated the diagnostic accuracy of RDTs in children suspected of severe malaria, one in Mozambique and Tanzania [4] and another in Tanzania [5]. The former compared a $P f$-pLDH with a PfHRP2-detecting RDT: they observed a significant lower sensitivity for the $P f$-pLDH-detecting RDT (88.0 vs $94.0 \%)$, especially at low $(<1,000 / \mu \mathrm{l})$ parasite densities. Of note however, the test used (Optimal-IT) is a multistep RDT with reported lower sensitivities compared to more recent one-step $P f$-pLDH-detecting RDT products $[8,19]$. In the present study there was excellent sensitivity of $P f$-pLDH detection, also at low parasite densities. Only one sample was missed by $P f$-pLDH detection and this sample had a parasite density of $62 / \mu \mathrm{l}$, which is below the detection threshold of routine microscopy [20].

Faint test lines, which are prone to be disregarded by health workers [21-23], only occurred in 0.5 and $2.4 \%$ of the true positive $P f \mathrm{HRP} 2$ and $P f$-pLDH lines, respectively. The co-presence of weak PfHRP2 and strong $P f$ pLDH lines at high parasite densities $(n=7)$, may be caused by the prozone effect (false negative/low test lines due to an antigen excess [24]), but was presently not further assessed. Only PfHRP2-detecting RDTs are affected by the prozone effect, not Pf-pLDH [24,25]. The degree to which RDTs are affected by prozone is product dependent and in some products PfHRP2 lines may be completely absent [24], leading to false negative results. The weak line intensities at high parasite densities are also of concern, as they may be considered as nonsevere disease $[23,26]$.

\section{Specificity}

When interpreting specificity, several factors should be addressed: first, although in the present study slides were double-read by experienced microscopists, very low parasite densities may have been missed. Next, exclusive presence of gametocytes was considered as $P$. falciparum negative (as they do not cause clinical infection) but they produce PfHRP2 and Pf-pLDH $[27,28]$ explaining the apparent false positive results. For the purpose of this study PCR was not considered as reference method, because it may detect submicroscopic infections (reflecting asymptomatic carriage) which do not explain clinical symptoms [29]. The false positive $P f$-pLDH lines observed in the present study can in part be explained by a (ongoing and partly) treated malaria infection from the previous days as $P f$-pLDH becomes negative in a median of two to seven days after start of effective treatment $[30,31]$. This was presently supported by its association with a history of recent anti-malarial treatment. In addition, there were three false positive $P f$-pLDH results for which both PfHRP2 and PCR were negative. Possible explanations may be cross-reaction with pLDH produced by $P$. ovale $(\mathrm{n}=2)$ or other interfering factors [32].

The interpretation of false positive PfHRP2 lines is more complex. The most common cause of false positive PfHRP2 results, especially in high-transmission areas, is PfHRP2 persistence. Other possibilities, though rare, are non-specific bindings or interference with other immunological or infectious factors, such as the rheumatoid factor, hepatitis C, schistosomiasis, toxoplasmosis, dengue, leishmaniasis, Chagas disease and human African trypanosomiasis [19,33-36].

For the PCR negative samples, it can assumed that the false positive PfHRP2 lines were due to past infection, approximately two to six weeks ago. For the PCR positive samples, the subsequent question arises whether the children were actually suffering from malaria at the time of sampling and had negative microscopy because of recently (<two days) started anti-malarial treatment (ongoing infection) [37] or whether it was a recently cleared infection with the child now suffering from another disease. Indeed, microscopy turns negative within one to two days after start of artemisinin-based combination therapy (ACT) [38], but the time of PCR to become negative after start of treatment has not yet been studied, although one study reported that upon completion 
of supervised ACT treatment a third of children were still positive by real time PCR [39]. As the proportion of positive PCR results among false positive PfHRP2 samples was high in the present study, it can be assumed that at least part of the false-positive PfHRP2 lines can be explained by ongoing and partly treated infection, especially in those samples that showed false positive $P f$ pLDH results as well.

Prior use of anti-malarial treatment (either by selfmedication or prescription) reflects real-life situation in malaria-endemic settings. To know if the child is actually suffering from malaria, an ideal RDT should be able to differentiate ongoing infection from a previously currently cured episode of infection, but PfHRP2 is not capable of doing so. Pf-pLDH RDTs seem to be more promising in that respect as they turn negative in two to seven days, but future studies should assess their evolution over time after start of treatment.

\section{Influence of age and season on specificity}

The low PfHRP2 specificity in the rainy and post-rainy season compared to the dry season has been observed before [40] and may be explained by malaria infection in the weeks prior to enrolment, as malaria transmission is high in these months. In addition, children may have had an actual infection and been (partly) treated before enrolment, which also explains the decreased Pf-pLDH specificity in the rainy and post-rainy season. For children aged one to five years, specificity for PfHRP2-detection was extremely low which may be ascribed to their high vulnerability to malaria, which was reflected by the high prevalence in this age group. The relationship has been observed before [5] while no such association was observed for $P f$-pLDH detection, probably due to the more rapid clearance from the blood stream [41-43].

\section{What if treatment is based on RDT results?}

In children with severe malaria it is crucial that the diagnosis is not missed: a negative RDT should safely exclude malaria. However, over diagnosis of malaria by diagnostic testing not only leads to a waste of antimalarial drugs but also increases the risk of ignorance of other possible life-threatening diseases, such as invasive bacterial infections, which is especially true for PfHRP2detecting RDTs. Even though WHO mentions to look for other causes of severe illness (including IBI) in the case of a positive RDT, this strategy is not yet clearly implemented in the diagnostic algorithm of Burkina Faso [11] and may be overlooked in daily reality, especially since tools for diagnosis of other diseases are lacking.

\section{Pf-pLDH versus PfHRP2-detecting RDTs}

The present data adds to the debate about $P f$-pLDH $v s$ PfHRP2-detecting RDTs. The previously reported lower sensitivity and lower heat stability of $P f$-pLDH-detecting RDTs appeared to be product dependent [19]. Unlike $P f H R P 2, P f$-pLDH-detection is not affected by the prozone effect [24], no gene deletions [15] or antigen polymorphisms [44-47] have been reported and it is rapidly cleared from the blood stream [41,42]. Especially because of its higher specificity, Pf-pLDH-detecting RDTs may be more useful in malaria-endemic areas. In addition it may be that Pf-pLDH-detecting RDTs are more cost-beneficial compared to presumptive diagnosis in high-transmission settings, which was not the case for PfHRP2-detecting RDTs as concluded by Bisoffi et al. [48]. This needs however to be further evaluated.

\section{Future perspectives}

To what extent can the present study findings be applied? First, an appropriate $P f$-pLDH-detecting RDT should be selected and assessed for its diagnostic accuracy and robustness in field studies. Currently only few $P f$-pLDH-detecting RDT products are available on the international market [32], and only one three-band RDT targeting Pf-pLDH and pan-pLDH and one two-band RDT targeting pan-pLDH fulfilled WHO criteria of good performance [49]. More emphasis should be on development and optimization of Pf-pLDH-detecting RDTs, and it should be evaluated how fast they become negative during anti-malarial treatment. If future field evaluations of $P f$-pLDH-detecting RDTs are satisfying, the recommendation of WHO to use PfHRP2-detecting RDTs in P. falciparum-endemic areas in sub-Saharan Africa [10] should be reconsidered. In the meantime, diagnostic algorithms should better highlight the possibility of invasive bacterial infections in spite of a positive RDT result.

\section{Conclusion}

Among children presenting with severe febrile illness in a region with seasonal hyperendemic malaria transmission, similar sensitivity but lower specificity of PfHRP2 compared to $P f$-pLDH-detection was observed. Specificity of PfHRP2 was lowest in the rainy season but PPV was highest in this season due to the high malaria prevalence. For each season and age group, the PPV of PfHRP2 was lower compared to Pf-pLDH. Part of the apparent false-positive PfHRP2 samples might however be due to parasite clearance after (incomplete or ongoing) treatment with anti-malarials at home or at the referring health centre. Further studies should assess the diagnostic accuracy and safety of an appropriate $P f$-pLDH-detecting RDT in field settings and its capacity to distinguish ongoing malaria from recently cleared infections. If satisfying, replacement of $P f \mathrm{HRP} 2$-detecting RDTs by $P f$-pLDHdetecting RDTs should be considered. 


\section{Abbreviations}

ACT: Artemisinin-based combination therapy; CRUN: Clinical research unit of Nanoro; EDTA: Ethylene diamine tetra-acetic acid; IBI: Invasive bacterial infections; ITM: Institute of Tropical Medicine; NPV: Negative predictive value; NTS: Non-typhoid Salmonella spp.; Pan-pLDH: Pan Plasmodium lactate dehydrogenase; PCR: Polymerase chain reaction; PfHRP2: P. falciparum Histidine-rich protein 2; Pf-pLDH: Plasmodium falciparum-specific parasite lactate dehydrogenase; pLDH: Parasite lactate dehydrogenase; PPV: Positive predictive value; RDT(s): Rapid diagnostic test(s); TBF: Thick blood film: WHO: World Health Organization.

\section{Competing interests}

The authors declare that they have no competing interests.

\section{Authors' contributions}

JM, CVG, TH, and JJ conceived and designed the study. JM, GI and BK supervised patient inclusion. JM, PL and PG performed the laboratory analyses. JM and JJ analysed the data and drafted the manuscript. All authors read and approved the final manuscript.

\section{Acknowledgements}

We thank CRUN laboratory staff for their contribution and clinical staff of CMA and Nanoro Health District for their collaboration and for patient care. The work of JM was funded by "Steunfonds Marguerite-Marie Delacroix". For this study, JM received additional grants from the Fund for Scientific Research-Flanders (F.W.O.-Vlaanderen, Belgium), the Junior Mobility program of KU Leuven and McKinsey \& Company. The RDTs were provided for free by Standard Diagnostics. The funders had no role in study design, data collection and analysis, decision to publish, or preparation of the manuscript.

\section{Author details}

'Department of Clinical Sciences, Institute of Tropical Medicine, Nationalestraat 155, B 2000, Antwerp, Belgium. ${ }^{2}$ Centre for Molecular and Vascular Biology, University of Leuven, Leuven, Belgium. ${ }^{3}$ IRSS/Clinical Research Unit of Nanoro (CRUN), Nanoro, Burkina Faso. ${ }^{4}$ Paediatrics, University of Leuven, Leuven, Belgium.

Received: 11 October 2013 Accepted: 28 December 2013

Published: 13 January 2014

\section{References}

1. World Health Organization: Guidelines for the Treatment of Malaria. 2nd edition. Geneva: World Health Organization; 2010.

2. Abba K, Deeks JJ, Olliaro P, Naing CM, Jackson SM, Takwoingi Y, Donegan S, Garner P: Rapid diagnostic tests for diagnosing uncomplicated $P$. falciparum malaria in endemic countries. Cochrane Database Syst Rev 2011, Issue 7:CD008122.

3. D'Acremont V, Malila A, Swai N, Tillya R, Kahama-Maro J, Lengeler C, Genton B: Withholding antimalarials in febrile children who have a negative result for a rapid diagnostic test. Clin Infect Dis 2010, 51:506-511.

4. Hendriksen IC, Mtove G, Pedro AJ, Gomes E, Silamut K, Lee SJ, Mwambuli A, Gesase S, Reyburn H, Day NP, White NJ, von Seidlein L, Dondorp AM: Evaluation of a PfHRP2 and a pLDH-based rapid diagnostic test for the diagnosis of severe malaria in 2 populations of African children. Clin Infect Dis 2011, 52:1100-1107.

5. Mtove G, Nadjm B, Amos B, Hendriksen IC, Muro F, Reyburn H: Use of an HRP2-based rapid diagnostic test to guide treatment of children admitted to hospital in a malaria-endemic area of north-east Tanzania. Trop Med Int Health 2011, 16:545-550.

6. Mayxay M, Pukrittayakamee S, Chotivanich K, Looareesuwan S, White NJ: Persistence of Plasmodium falciparum HRP-2 in successfully treated acute falciparum malaria. Trans R Soc Trop Med Hyg 2001, 95:179-182.

7. World Health Organization: Malaria Rapid Diagnostic Test Performance; Results of WHO product testing of malaria RDTs: 4 (2012). Geneva: World Health Organization; 2012.

8. Heutmekers M, Gillet P, Maltha J, Scheirlinck A, Cnops L, Bottieau E, Van EM, Jacobs J: Evaluation of the rapid diagnostic test CareStart pLDH Malaria (Pf-pLDH/pan-pLDH) for the diagnosis of malaria in a reference setting. Malar J 2012, 11:204
9. Ratsimbasoa A, Randriamanantena A, Raherinjafy R, Rasoarilalao N, Menard D: Which malaria rapid test for Madagascar? Field and laboratory evaluation of three tests and expert microscopy of samples from suspected malaria patients in Madagascar. Am J Trop Med Hyg 2007, 76:481-485

10. World Health Organization: Good Practices for Selecting and Procuring Rapid Diagnostic Tests for Malaria. Geneva: World Health Organization; 2011.

11. Ministère de la sante Burkina Faso: Manuel de Formation Pour la Prise en Charge des cas de Paludisme au Niveau des Formations Sanitaires. Manuel du Participant. ; 2010.

12. Institut National de la Statistique et de la Démographie (INSD) et ICF International: Enquête Démographique et de Santé et à Indicateurs Multiples du Burkina Faso 2010. Calverton, Maryland, USA: INSD et ICF International; 2012.

13. Maltha J, Guiraud I, Kaboré B, Lompo P, Ley B, Van Geet C, Tinto H, Jacobs J: Frequency of severe malaria and invasive bacterial infections among children admitted to a rural hospital in Burkina Faso. PLoS One. in press.

14. World Health Organization: Basic Malaria Microscopy - Part I.Learner's Guide. 2nd edition. Geneva: World Health Organization; 2010.

15. Maltha J, Gamboa D, Bendezu J, Sanchez L, Cnops L, Gillet P, Jacobs J: Rapid diagnostic tests for malaria diagnosis in the Peruvian Amazon: impact of pfhrp2 gene deletions and cross-reactions. PLoS One 2012, 7:e43094.

16. Heutmekers M, Gillet P, Cnops L, Bottieau E, Van EM, Maltha J, Jacobs J: Evaluation of the malaria rapid diagnostic test SDFK90: detection of both PfHRP2 and Pf-pLDH. Malar J 2012, 11:359.

17. Cnops L, Van EM, Bottieau E, Jacobs J: Giemsa-stained thick blood films as a source of DNA for Plasmodium species-specific real-time PCR. Malar J 2010, 9:370.

18. Cnops $L$, Jacobs J, Van EM: Validation of a four-primer real-time $P C R$ as a diagnostic tool for single and mixed Plasmodium infections. Clin Microbiol Infect 2011, 17:1101-1107.

19. World Health Organization: Malaria Rapid Diagnostic Test Performance Results of WHO Product Testing of Malaria RDTs: Round 3 (2010-2011). Geneva: World Health Organization; 2011.

20. Milne LM, Kyi MS, Chiodini PL, Warhurst DC: Accuracy of routine laboratory diagnosis of malaria in the United Kingdom. J Clin Pathol 1994, 47:740-742.

21. Counihan H, Harvey SA, Sekeseke-Chinyama M, Hamainza B, Banda R, Malambo T, Masaninga F, Bell D: Community health workers use malaria rapid diagnostic tests (RDTs) safely and accurately: results of a longitudinal study in Zambia. Am J Trop Med Hyg 2012, 87:57-63.

22. Harvey SA, Jennings $L$, Chinyama M, Masaninga F, Mulholland K, Bell DR: Improving community health worker use of malaria rapid diagnostic tests in Zambia: package instructions, job aid and job aid-plus-training. Malar J 2008, 7:160.

23. Rennie W, Phetsouvanh R, Lupisan S, Vanisaveth V, Hongvanthong B, Phompida S, Alday P, Fulache M, Lumagui R, Jorgensen P, Bell D, Harvey S: Minimising human error in malaria rapid diagnosis: clarity of written instructions and health worker performance. Trans R Soc Trop Med Hyg 2007, 101:9-18.

24. Gillet P, Scheirlinck A, Stokx J, De WA, Chauque HS, Canhanga OD, Tadeu BT, Mosse CD, Tiago A, Mabunda S, Bruggeman C, Bottieau E, Jacobs J: Prozone in malaria rapid diagnostics tests: how many cases are missed? Malar J 2011, 10:166.

25. Gillet P, Mori M, Van Esbroeck M, Van Den EJ, Jacobs J: Assessment of the prozone effect in malaria rapid diagnostic tests. Malar J 2009, 8:271.

26. Asiimwe C, Kyabayinze DJ, Kyalisiima Z, Nabakooza J, Bajabaite M, Counihan H, Tibenderana JK: Early experiences on the feasibility, acceptability, and use of malaria rapid diagnostic tests at peripheral health centres in Uganda-insights into some barriers and facilitators. Implement Sci 2012, 7:5.

27. Oduola AM, Omitowoju GO, Sowunmi A, Makler MT, Falade CO, Kyle DE, Fehintola FA, Ogundahunsi OA, Piper RC, Schuster BG, Milhous WK: Plasmodium falciparum: evaluation of lactate dehydrogenase in monitoring therapeutic responses to standard antimalarial drugs in Nigeria. Exp Parasitol 1997, 87:283-289.

28. Hayward RE, Sullivan DJ, Day KP: Plasmodium falciparum: histidine-rich protein II is expressed during gametocyte development. Exp Parasitol 2000, 96:139-146.

29. World Health Organization: Disease Surveillance for Malaria Elimination: An Operational Manual. Geneva: World Health Organization; 2012.

30. Ashley EA, Touabi M, Ahrer M, Hutagalung R, Htun K, Luchavez J, Dureza C, Proux S, Leimanis M, Lwin MM, Koscalova A, Comte E, Hamade P, Page AL, 
Nosten F, Guerin PJ: Evaluation of three parasite lactate dehydrogenase-based rapid diagnostic tests for the diagnosis of falciparum and vivax malaria. Malar J 2009, 8:241.

31. Gerstl S, Dunkley S, Mukhtar A, De Smet M, Baker S, Maikere J: Assessment of two malaria rapid diagnostic tests in children under five years of age, with follow-up of false-positive pLDH test results, in a hyperendemic falciparum malaria area. Sierra Leone. Malar J 2010, 9:28.

32. Maltha J, Gillet $P$, Jacobs J: Malaria rapid diagnostic tests in endemic settings. Clin Microbiol Infect 2013, 19:399-407.

33. Iqbal J, Sher A, Rab A: Plasmodium falciparum histidine-rich protein 2-based immunocapture diagnostic assay for malaria: cross-reactivity with rheumatoid factors. J Clin Microbiol 2000, 38:1184-1186.

34. Maltha J, Gillet P, Cnops L, Bottieau E, Van Esbroeck M, Bruggeman C, Jacobs J: Evaluation of the rapid diagnostic test SDFK40 (Pf-pLDH/pan-pLDH) for the diagnosis of malaria in a non-endemic setting. Malar J 2011, 10:7.

35. Leshem E, Keller N, Guthman D, Grossman T, Solomon M, Marva E, Schwartz E: False-positive Plasmodium falciparum histidine-rich protein 2 immunocapture assay results for acute schistosomiasis caused by Schistosoma mekongi. J Clin Microbiol 2011, 49:2331-2332.

36. Gillet P, Mumba ND, Lukuka A, Kande V, Atua B, van GJ, Muyembe JJ, Jacobs J, Lejon $\mathrm{V}$ : False positivity of non-targeted infections in malaria rapid diagnostic tests: the case of human african trypanosomiasis. PLOS Negl Trop Dis 2013, 7:e2180.

37. World Health Organization: Management of Severe Malaria: A Practical Handbook. Geneva: World Health Organization; 2012.

38. Agarwal A, McMorrow M, Onyango P, Otieno K, Odero C, Williamson J, Kariuki S, Kachur SP, Slutsker L, Desai M: A randomized trial of artemether-lumefantrine and dihydroartemisinin-piperaquine in the treatment of uncomplicated malaria among children in western Kenya. Malar J 2013, 12:254.

39. Beshir KB, Sutherland CJ, Sawa P, Drakeley CJ, Okell L, Mweresa CK, Omar SA, Shekalaghe SA, Kaur H, Ndaro A, Chilongola J, Schallig HD, Sauerwein RW, Hallett RL, Bousema T: Residual Plasmodium falciparum parasitemia in Kenyan children after artemisinin-combination therapy is associated with increased transmission to mosquitoes and parasite recurrence. J Infect Dis 2013, 208:2017-2024.

40. Bisoffi Z, Sirima SB, Menten J, Pattaro C, Angheben A, Gobbi F, Tinto H, Lodesani C, Neya B, Gobbo M, Van den Ende J: Accuracy of a rapid diagnostic test on the diagnosis of malaria infection and of malaria-attributable fever during low and high transmission season in Burkina Faso. Malar J 2010, 9:192.

41. Fogg C, Twesigye R, Batwala V, Piola P, Nabasumba C, Kiguli J, Mutebi F, Hook C, Guillerm M, Moody A, Guthmann JP: Assessment of three new parasite lactate dehydrogenase (pan-pLDH) tests for diagnosis of uncomplicated malaria. Trans R Soc Trop Med Hyg 2008, 102:25-31.

42. Iqbal J, Siddique A, Jameel M, Hira PR: Persistent histidine-rich protein 2, parasite lactate dehydrogenase, and panmalarial antigen reactivity after clearance of Plasmodium falciparum monoinfection. J Clin Microbio/ 2004 42:4237-4241.

43. Makler MT, Palmer CJ, Ager AL: A review of practical techniques for the diagnosis of malaria. Ann Trop Med Parasitol 1998, 92:419-433.

44. Baker J, McCarthy J, Gatton M, Kyle DE, Belizario V, Luchavez J, Bell D, Cheng Q: Genetic diversity of Plasmodium falciparum histidine-rich protein 2 (PfHRP2) and its effect on the performance of PfHRP2-based rapid diagnostic tests. J Infect Dis 2005, 192:870-877.

45. Baker J, Ho MF, Pelecanos A, Gatton M, Chen N, Abdullah S, Albertini A, Ariey F, Barnwell J, Bell D, Cunningham J, Djalle D, Echeverry DF, Gamboa D, Hii J, Kyaw MP, Luchavez J, Membi C, Menard D, Murillo C, Nhem S, Ogutu B, Onyor P, Oyibo W, Wang SQ, McCarthy J, Cheng Q: Global sequence variation in the histidine-rich proteins 2 and 3 of Plasmodium falciparum: implications for the performance of malaria rapid diagnostic tests. Malar J 2010, 9:129.

46. Mariette N, Barnadas C, Bouchier C, Tichit M, Menard D: Country-wide assessment of the genetic polymorphism in Plasmodium falciparum and Plasmodium vivax antigens detected with rapid diagnostic tests for malaria. Malar J 2008, 7:219.

47. Kumar N, Singh JP, Pande V, Mishra N, Srivastava B, Kapoor R, Valecha N, Anvikar AR: Genetic variation in histidine rich proteins among Indian Plasmodium falciparum population: possible cause of variable sensitivity of malaria rapid diagnostic tests. Malar J 2012, 11:298.

48. Bisoffi Z, Sirima SB, Meheus F, Lodesani C, Gobbi F, Angheben A, Tinto H, Neya B, Van den Ende K, Romeo A, Van den Ende J: Strict adherence to malaria rapid test results might lead to a neglect of other dangerous diseases: a cost benefit analysis from Burkina Faso. Malar J 2011, 10:226.

49. The global fund: List of Rapid Diagnostic Test (RDT) Kits for Malaria Classified According to the Global Fund Quality Assurance Policy. Version 8; 2013. www. theglobalfund.org/documents/psm/PSM_QADiagnostics_Malaria_list/. Accessed 8/ 9/2013.

doi:10.1186/1475-2875-13-20

Cite this article as: Maltha et al.: Accuracy of PfHRP2 versus Pf-pLDH antigen detection by malaria rapid diagnostic tests in hospitalized children in a seasonal hyperendemic malaria transmission area in Burkina Faso. Malaria Journal 2014 13:20.

\section{Submit your next manuscript to BioMed Central and take full advantage of:}

- Convenient online submission

- Thorough peer review

- No space constraints or color figure charges

- Immediate publication on acceptance

- Inclusion in PubMed, CAS, Scopus and Google Scholar

- Research which is freely available for redistribution

Submit your manuscript at www.biomedcentral.com/submit
C) Biomed Central 